\title{
CoMIC, the hidden dynamics of mitochondrial inner compartments
}

\author{
Bongki Cho ${ }^{1} \&$ Woong Sun ${ }^{2, *}$ \\ ${ }^{1}$ Department of Brain \& Cognitive Sciences, Daegu Gyeongbuk Institute of Science and Technology, Daegu 42988, ${ }^{2}$ Department of \\ Anatomy, Korea University College of Medicine, Seoul 02841, Korea
}

\begin{abstract}
Mitochondria have evolutionarily, functionally and structurally distinct outer- (OMM) and inner-membranes (IMM). Thus, mitochondrial morphology is controlled by independent but coordinated activity of fission and fusion of the OMM and IMM. Constriction and division of the OMM are mediated by endocytosis-like machineries, which include dynamin-related protein 1 with additional cytosolic vesicle scissoring machineries such as actin filament and Dynamin 2. However, structural alteration of the IMM during mitochondrial division has been poorly understood. Recently, we found that the IMM and the inner compartments undergo transient and reversible constriction prior to the OMM division, which we termed CoMIC, Constriction of $\underline{M}$ itochondrial Inner $\underline{\text { Compartment. In this short }}$ review, we further discuss the evolutionary perspective and the regulatory mechanism of CoMIC during mitochondrial division. [BMB Reports: Perspective 2017; 50(12): 597-598]
\end{abstract}

Mitochondria play essential roles in the life of cells as a cellular powerhouse to produce ATP, a storage center to store calcium or other vital ions, and a biochemical reactor to generate specific lipids. These essential mitochondrial functions are closely associated with mitochondrial morphology (Chan (2012) Annual review of genetics 46, 265-287). Upon changes in the cellular demands for cellular functions, mitochondria alter their morphology and distribution within cells. Accordingly, failure of these essential morphological responses is

*Corresponding author. E-mail: woongsun@korea.ac.kr

https://doi.org/10.5483/BMBRep.2017.50.12.154

Received 8 August 2017

Keywords: Calcium, Constriction, Division, Drp1, Mitochondria

Abbreviations: Drp1, Dynamin-related protein 1; ER, endoplasmic reticulum; FRET, fluorescence resonance energy transfer; IMM, mitochondrial inner membrane; Mfn1/2, Mitofusin 1/2; OMM, mitochondrial outer membrane; Opal, Optic atrophy 1

Perspective to: Cho, B. et al., 2017, Constriction of the mitochondrial inner compartment is a priming event for mitochondrial division, Nature Communications, 8, 15754. doi: 10.1038/ncomms 15754 . associated with many diseases, which further highlights the importance of regulation of mitochondrial structure. Mitochondrial morphology is determined by dynamic equilibrium between fusion and division, which are mediated by large GTPases. Because the OMM and the IMM have vastly different biochemical and molecular properties, mitochondrial morphogenesis requires distinct but coordinated machineries for each membrane. In mammalian cells, mitochondrial fusion is completed by OMM fusion and subsequent IMM fusion, which are mediated by $M f n 1 / 2$ and Opa1, respectively. Unlike mitochondrial fusion, mitochondrial division has been believed to be solely driven by constriction of mitochondria from the outside. Drp1 is a principal molecule which translocates from the cytosol to the OMM, oligomerizes, and finally constricts to divide mitochondria. The oligomerization of homogenous Drp1 molecules forms a spiral-like shape with a very narrow inner diameter $(\sim 89 \mathrm{~nm})$ to initiate or to complete mitochondrial division (Mears et al. (2011) Nat Struct Mol Biol 18, 20-26). Thus, mitochondrial division requires complementary machineries: actin assembly for promoting initial constriction at the ER-mitochondria contact site (Korobova et al. (2013) Science $339,464-467)$, and the involvement of Dynamin 2 for executing the final division after Drp1-mediated constriction (Lee et al. (2016) Nature 540, 139-143). These mechanisms are highly similar to endocytosis of vesicles, indicating that mitochondrial division machineries for the OMM may evolutionarily originate from dynamin-mediated endocytosis.

According to the endosymbiosis theory, the IMM is evolutionarily derived from the cell membrane of a proteobacteria-like endosymbiont (Kuroiwa et al. (2008) Int Rev Cell Mol Biol 271, 97-152). Prokaryotic cell division utilizes intracellular Z-ring, which is formed by the tubulin-like protein FtsZ. Consistently, mitochondria of some primitive eukaryotes have FtsZ orthologues and exhibit a Z-ring-like structure in the mitochondrial matrix before the formation of the outer division ring. Unlike primitive eukaryotes, neither FtsZ-like proteins nor Z-ring-like structures have been found in mammalian cells, and the division machinery in the mitochondrial inner compartment has been believed to be lost in mammalian cells. However, we recently found interesting morphological dynamics of mitochondrial inner compartments which exhibit transient transition of their string-like morphology to beads-on- 
a-string form, which we termed CoMIC. We believe that CoMIC compensates for the deficiency of FtsZ and Z-ring in higher eukaryotes.

CoMIC appears to be a priming event for efficient mitochondrial division, although CoMIC per se cannot execute the division of mitochondria. CoMIC is a transient and reversible event ( $\sim 1.5$ minutes per a 10-minute period), and its occurrence is strongly associated with subsequent mitochondrial division. In addition, constriction sites of the CoMIC are spatially coupled with putative Drp1-driven mitochondrial division sites. Furthermore, the prevention of Drp1 activity dramatically augmented the cyclic nature of $\mathrm{COMIC}$, indicating that execution of mitochondrial division may terminate the CoMIC cycle. Conversely, inhibition of CoMIC suppressed Drp1-dependent mitochondrial division. These findings suggest that CoMIC is a priming event for mitochondrial division (Diagram 1). Intramitochondrial influx of calcium from ER directly promoted the CoMIC, leading to two synergistic processes; 1) intramitochondrial influx of potassium through a calcium-dependent potassium channel, and 2) production of short Opa1 localizing in the intermembrane space by Oma1-mediated cleavage. These processes cause osmotic swelling of mitochondrial matrix and reorganization of IMM-OMM tethering, respectively. This mode is similar to cell division by mechanical budding in some FtsZ-less prokaryotes (Miyagishima et al. (2014) Front Plant Sci 5, 459), implying that the CoMIC is evolutionarily derived from an ancient machinery. It is unclear whether there are any yet-to-be-found molecule(s) structurally ordering the CoMIC via oligomerization, or whether osmotic pressures with local rearrangement of cristae-forming complex are sufficient to drive the CoMIC. In addition, it is a mystery how the constriction phase in CoMIC is relaxed and a new round of CoMIC is initiated in the absence of Drp1-dependent execution of mitochondrial division.

Recent live-cell imaging techniques have revealed 'physiological flashes' in mitochondria, which are transient fluctuations of mitochondrial membrane potential, superoxide, $\mathrm{pH}$ and oxidation (Wang et al. (2016) Antioxid Redox Signal 25, 534-549). Interestingly, these mitochondrial flashes are also associated with CoMIC (Lee et al. (2014) J Biol Chem 289, 11862-11872; Breckwoldt et al. (2014) Nat Med 20, 555-560). Flash of intra-mitochondrial calcium can promote mitochondrial production of ATP and ROS. Notably, intra-mitochondrial calcium indirectly promoted mitochondrial division as well as CoMIC. Thus, intra-mitochondrial calcium might control the coordination of structural and physiological flashes in mitochondria. To further understand the physiological role of CoMIC, cell-type dependent contribution of mitochondrial

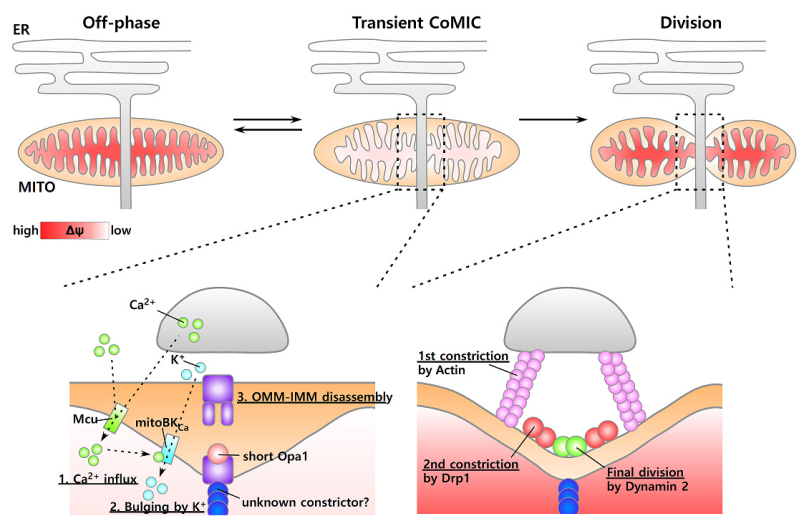

Diagram 1. Schematic model for CoMIC and mitochondrial division. COMIC is initiated by mitochondrial influx of calcium, leading to mitochondrial bulging and OMM-IMM disassembly which are mediated by potassium and short Opa1, respectively. Mitochondrial division is completed by actin-mediated initial constriction, Drp1-mediated second constriction, and Dynamin 2-mediated final division.

functions should be considered. Although the presence of CoMIC is evident in most cell types including embryonic stem cells, fibroblasts, neurons and hepatocytes, the extent of CoMIC appears to depend on the cell type. Especially, neuronal cells exhibit strongest basal CoMIC activity, compared to other cell types.

According to our current model, the distance between the OMM and the IMM should be focally changed during CoMIC. We found that FRET signal of two fluorescent proteins (one in the OMM and the other in the mitochondrial matrix) was detectable and dynamically changed in consistency with our model. Considering that FRET occurs only in very close proteins within 1-10 nm distance, this result suggests that the OMMIMM association in live cells is close enough to generate FRET, and it is much closer than that previously speculated by electron microscopy (12-25 nm) (Reichert et al. (2002) Biochim Biophys Acta 1592, 41-49). We also found that the OMM-IMM distance is highly dynamic with FRET, even in the absence of CoMIC, and the functional significance of this new type of structural dynamics should be explored further.

\section{ACKNOWLEDGEMENTS}

This research was supported by the National Research Foundation of Korea (NRF-2016R1A6A3A11930298) to B.C, and (NRF-2015M3C7A1028790 and NRF-2012M3A9C6049933) to W.S. 